\section{El San Juan de Dios y las trabajadoras: la vida social de un hospital y la crisis de la salud en Colombia}

\author{
The San Juan de Dios \\ Hospital and its workers: the \\ social life of a hospital and \\ the health crisis in Colombia
}

Andrés Góngora

Investigador asociado, Centro de Estudios Sociales/ Universidad Nacional de Colombia; Núcleo de Pesquisas em Cultura e Economia/Programa de Pós-Graduação em Antropologia Social/Universidade Federal do Rio de Janeiro. Calle 44 No. 45-67 - Bloque B5 y B6 Bogotá - Colombia

algongoras@unal.edu.co
GÓNGORA, Andrés. El San Juan de Dios y las trabajadoras: la vida social de un hospital y la crisis de la salud en Colombia. História, Ciências, SaúdeManguinhos, Rio de Janeiro, v.24, n.4, out.-dez. 2017, p.953-968.

Resumen

Este artículo describe algunos eventos significativos de los 15 años de lucha (1999-2014) de un grupo de mujeres por la reactivación del Hospital San Juan de Dios y por continuar siendo trabajadoras después de las reformas neoliberales en Colombia. La etnografía presentada propone herramientas para la comprensión de las dimensiones morales forjadas a partir de un conflicto por el reconocimiento de derechos y demuestra que el hospital, más que un conjunto arquitectónico, es un idioma activado por las trabajadoras para afirmar su humanidad, un vehículo para la elaboración simbólica de una causa y un medio para entender la construcción de colectividades a partir de gramáticas emocionales.

Palabras clave: trabajo; antropología; hospitales; salud pública.

\section{Abstract}

This article describes some significant events of the fifteen years of struggle (1999-2014) of a group of women for the reactivation of the San Juan de Dios Hospital and to stay on as workers after the neoliberal reforms in Colombia. The ethnography presented proposes tools for the understanding of the moral dimensions forged from a conflict for the recognition of rights and shows that the hospital, more than being an architectural complex, is a language activated by the workers to affirm their humanity, a vehicle for the symbolic elaboration of a cause and a means to understand the construction of collectives based on emotional terminology.

Keywords: work; anthropology; hospitals; public health. 
E n 1999 fueron suspendidos los salarios a 2.480 empleados del Hospital San Juan de Dios. Desde entonces han sido sometidos a toda clase de atropellos por parte de distintos agentes de la administración pública encargados de darle solución a su "problema laboral", entendido como la causa principal de su sufrimiento y del cese de operaciones que hoy mantiene en un proceso de deterioro progresivo al primer y más importante hospital público de Colombia. ${ }^{2}$ En general este tipo de conflictos son interpretados, desde el punto de vista del derecho, dejando de lado las experiencias de los actores que alimentan y sostienen las causas políticas. Este artículo recoge buena parte del diálogo con un grupo de trabajadoras del hospital (principalmente enfermeras y funcionarias del área de servicios generales) e intenta aportar a la comprensión de este proceso social desde una perspectiva antropológica. ${ }^{3}$ Para esto propongo explorar etnográficamente posibilidades de agencia que permiten relativizar los límites semánticos impuestos por la racionalidad jurídica y pensar, como lo propone Myriam Jimeno (2010) en la producción de "verdades sociales". Considero que esta aproximación puede mejorar las descripciones que sitúan a los actores sociales como "víctimas inermes" e impiden ver otras formas de producción de valor. Siguiendo a Das y Poole (2004), defiendo la necesidad de pensar la agencia más allá de los "actos de resistencia" (y su correlato, la fetichización del "Estado") y estudiar los modos por medio de los cuales los límites conceptuales del "Estado" son extendidos y rehechos para asegurar la supervivencia y buscar justicia. Esto implica tomar distancia de la orientación predominantemente doctrinaria de la racionalidad jurídica, centrada en deliberaciones y fallos, para examinar "las disidencias, los planos morales y las estrategias para hacer valer posiciones o para ver reconocidas trayectorias y esfuerzos personales y colectivos frente a letra de la ley" (Vianna, 2014, p.16). Se trata de transitar hacia un análisis simbólico de los derechos (Cardoso de Oliveira, 2010), prestando atención a la trama de actores y agencias comprometidos con una causa, a los performances y materiales usados para darle verosimilitud y a la cosmología política obliterada por la rutinización o naturalización de los procedimientos burocráticos.

Varias de las trabajadoras del "San Juan" decidieron quedarse permanentemente en el hospital hasta obtener una respuesta a sus demandas (respeto de la convención colectiva, ${ }^{4}$ pago de todos los salarios atrasados desde 1999, indemnizaciones por daños y perjuicios sufridos durante el proceso, entre otras). Para ellas el "Estado" no es ese "socio amigable" con el cual las comunidades trabajan mancomunadamente en el desarrollo de políticas públicas, por el contrario, es el responsable de su desgracia y al mismo tiempo, la única entidad con la autoridad moral para restablecer los agravios padecidos. De manera análoga al concepto teológico de teodicea, conjunto de explicaciones para entender el origen del mal en un mundo divinamente ordenado (Weber, 1993), las protagonistas de esta historia construyen su propia "teodicea secular" (Hertzfel, 1993; Das, 2008) para intentar entender la perversidad de las instituciones de la administración pública que les han conculcado sus derechos y al tiempo cultivar la esperanza de que algún ente impartidor de justicia, nacional o internacional, les reconozca, en representación de la "verdadera democracia", su estatuto como trabajadoras. Ahora bien, esta teodicea secular, este amor y odio por todo lo que representa esa abstracción mistificada denominada "Estado" (Foucault, 2007, p.137) se ve reflejada en las prácticas 
cotidianas de lucha y en la manera en que esta "comunidad" define el trabajo, la salud, lo público, el cuidado y el propio hospital, que como se verá a lo largo de estas páginas, es conceptualizado como una persona moral. Hay pues un límite trazado cotidianamente entre las exigencias que pueden y deben ser dichas y las reclamaciones consideradas demasiado polémicas, fuera de lugar (situación recurrente en las contiendas por derechos) o incluso subversivas y en ese sentido, enemigas del "Estado". No se trata aquí, como lo han notado varios autores de fronteras definidas (Das, Poole, 2004; Jeganathan, 2004), sino, por el contrario, de circunscripciones porosas que definen los límites pantanosos del "Estado".

Dadas las dimensiones y el objetivo del presente artículo, relataré, brevemente, los principales eventos de los últimos años de lucha narrados desde el punto de vista de las trabajadoras para rastrear las condiciones de posibilidad y las gramáticas emocionales relativas a la construcción de una causa (Boltanski, 1993; Jimeno, 2010). De igual manera, intentaré describir algunos artefactos (Riles, 2006) para explorar la capacidad creativa y la agencia de este grupo de mujeres dedicadas a defender el Hospital San Juan de Dios. De un lado están los documentos, (recopilados, clasificados, estudiados y organizados para reconstruir la vida social del hospital) que las trabajadoras usan para trazar una narrativa particular que cuestiona el tiempo histórico ensamblado por los agentes heterogéneos que encarnan al "Estado" y, por otro lado, la red de personas, significados, memorias y objetos que componen la persona moral que las trabajadoras denominan "el hospital" o más coloquialmente "el San Juan". El análisis de estos artefactos apunta a una etnografía de las luchas en clave de tensiones espacio-temporales y semánticas (Feldman, 1991) localizadas en el proceso de deformación y reformación de las esferas material y experiencial de la vida.

El texto está dividido en dos secciones. La primera comprende la descripción de dos situaciones etnográficas presentadas con el ánimo de situar las perspectivas, antagónicas en muchos sentidos, de tres mujeres que representan a las facciones en que estaba dividido el grupo de trabajadoras del hospital San Juan de Dios. En la segunda sección se analiza el trabajo de estas mujeres para mantener viva la causa, destacando su papel como generadoras de conocimiento histórico y jurídico: el uso, producción y circulación de artefactos (dentro de los cuales se destacan documentos, edificios e instrumentos hospitalarios), los conflictos internos generados a lo largo de más de una década de lucha, las narrativas por medio de las cuales interpretan su experiencia y sustentan su lugar en el mundo y las implicaciones de la teoría construida por las trabajadoras según la cual el "San Juan es un sujeto de derechos".

\section{Trabajando en la causa}

Durante una de las marchas por el derecho a la salud, realizada durante el primer semestre de 2013, una de las trabajadoras del Hospital San Juan de Dios que para fines etnográficos llamaremos Clara, ${ }^{5}$ pronunció la siguiente arenga:

Compañeros y compañeras:

¡El gobierno nacional está en la obligación de reactivar el Hospital San Juan de Dios ya! Es deber de toda la Nación, no solo de Bogotá sino de Colombia entera, entrar en la lucha. ¡Arranquemos el San Juan de las garras al gobierno para que no se lo robe! ¡Recuperemos lo que es nuestro, que nos lo devuelvan, que le devuelvan al Hospital 
más antiguo de Colombia todo lo que le han quitado, desde el legado del José Joaquín $\operatorname{Vargas}^{6}$ del año 1957 hasta la fecha!

No nos engañemos, nunca hubo una crisis. Todo fue una pantomima que los gobiernos de turno generaron para poderse robar el San Juan, para que las EPS [aseguradoras] y todas esas mafias se pudieran apoderar de él. Y ellos se la cranearon [sic] bien. Dejaron al Hospital en cuidados intensivos, mercantilizaron la salud y todo les prosperó y les ha dado resultado hasta la fecha. Pero no nos han vencido.

Nosotros hemos estado todos los días desde el año 99 hasta la fecha. Cuando teníamos nuestros salarios trabajábamos cumpliendo con un horario como cualquier empleado, pero desde el año 99 algunos decidimos quedarnos aquí las 24 horas sin recibir una moneda. Por eso invitamos al pueblo colombiano diciéndole a viva voz: ¡Qué nuestro hospital es la cuna de la medicina! ¡Qué nuestro hospital salvó muchísimas vidas! ¡Qué le devolvamos la mano que nos brindó! ¡Qué le demos el oxígeno que necesita para que siga viviendo! ¡La salud es un derecho, no dejemos que el gobierno nos la arrebate! ¡No dejemos que las mafias se enriquezcan con cada muerto que ponemos!

Dos meses antes de la marcha en que este discurso fue promulgado, nos reunimos para analizar parte de las pruebas materiales usadas por las trabajadoras para sustentar su causa. Gladys estaba sentada en el comedor de la casa que nos servía como sede, intentando organizar un cartapacio de papeles que cuentan la historia del Hospital San Juan de Dios, el más antiguo y prestigioso de Colombia. Estos papeles, la gran mayoría fotocopias que Gladys guarda celosamente y que lleva consigo la mayor parte del tiempo, tienen diferentes particularidades formales, estéticas, administrativas y están datados en periodos que abarcan buena parte de la historia colonial y republicana del país. Algunos son manuscritos de los siglos XVII y XVIII, redactados por autoridades coloniales; otros fueron elaborados por jerarcas de la Iglesia católica e instituciones de beneficencia y otros por hombres ricos que decidieron dejar "legados" para el bien público. Sobre la mesa estaban también documentos más recientes, redactados por instituciones de la administración pública del Estado colombiano que abarcan principalmente las décadas de 1980, 1990 y los primeros años del siglo XXI; los primeros confeccionados en máquinas de escribir, los últimos en computadores, pero todos debidamente sellados y foliados, con señales visibles de haber sido manipulados por muchos lectores. Entre estos documentos hay leyes, fallos, decretos, inventarios, acuerdos, cartas de protesta, denuncias públicas y acciones de tutela. Para Gladys, estos papeles constituyen las pruebas de una injusticia cometida en contra de un grupo de trabajadores y trabajadoras del Hospital San Juan de Dios que fueron despedidos ilegalmente hace más de una década. Durante ese periodo de tiempo, varios fallecieron, otros desistieron y buscaron nuevos trabajos, casi siempre borrando de sus currículos su pasado sindicalista que los hacía aparecer como una amenaza y les impedía conseguir empleo, pero un grupo, cada vez más reducido y constituido mayoritariamente por mujeres, había permanecido políticamente activo, luchando por los derechos de los trabajadores (pensiones, salariaros atrasados, indemnizaciones, restablecimiento de daños morales) y la defensa de "lo público" (o mejor, del carácter público del hospital y en general de la salud). Gladys asegura que en esos documentos se encuentra una verdad que no ha podido salir a la luz debido a los intereses de personas poderosas que quieren apropiarse del hospital, privatizarlo y usufructuarlo para su propio beneficio, en contra de su esencia, de la misión caritativa para la cual fue creado desde épocas coloniales y 
de su historia, que es también la historia de la medicina en Colombia. Desde el punto de vista jurídico, el caso de Gladys y de varias de sus compañeras no presentaba muchas posibilidades pues en varias oportunidades las "instancias competentes" de la administración pública habían fallado en su contra; por tal motivo Gladys aseguraba que la lucha debería centrarse en defender el hospital, y más aún, en acudir a instancias internacionales para hacer que el hospital sea declarado "sujeto de derechos", siguiendo el ejemplo de la Constitución Boliviana "que le otorgó prerrogativas específicas a la Pacha Mama y al Agua". En 1999, Gladys y otras personas, que ella denomina "la base" (empleados que desempeñaban cargos administrativos y de servicios generales), habían decidido permanecer en su lugar de trabajo y esto implicó "tomarse el San Juan" y, ante la inminente falta de trabajo y de recursos económicos, irse a vivir con sus familias en varios de los pabellones.

Nuestra reunión tenía dos objetivos: uno técnico y otro estratégico. Comenzar a organizar los documentos arriba descritos y mediar entre las distintas facciones en que estaba dividido el grupo de las trabajadoras del San Juan, propiciando un encuentro entre Gladys y Ligia (la tercera mujer cuya historia nos ayudará a entender el devenir de esta causa). Un par de horas después de iniciado el encuentro, entró en el recinto una mujer vestida de negro, notablemente abatida, que se presentaba como una de las enfermeras jefe del Hospital San Juan de Dios. Rápidamente entendí que el luto se debía al fallecimiento reciente de su mejor amiga y compañera de causa, otra trabajadora que se había ido sin ver la reapertura del San Juan. Ligia hacia parte de un grupo de profesionales que no permanecían todo el día en el hospital, pero que, no obstante, habían estado los mismos 15 años luchando por sus derechos como trabajadoras y por la defensa de "lo público". Cuando los pacientes dejaron de llegar, pues desde el punto de vista de las trabajadoras el hospital nunca ha estado cerrado, estas profesionales permanecieron "cuidándolo", preparando el instrumental médico, aseándolo, haciendo que se mantuviera en las mejores condiciones; el hospital, dice Ligia, aguardaba con los "brazos abiertos" el retorno de los pacientes y de los estudiantes que lo llenaban de vida. Pero esto no significaba que el hospital estuviese muerto, por el contario, gran parte del trabajo de estas mujeres se concentró en contradecir argumentos de periodistas, actores institucionales, académicos, políticos e incluso artistas plásticos que hablaban del San Juan como un ente agonizante, como un ser deteriorado, suspendido en el tiempo, repleto de objetos de otra época, debilitado por la desidia y la osadía de algunas personas que habían decidido invadirlo y convertirlo en lugar de residencia.

Pese a las diferencias internas, los relatos de Gladys, Ligia y Clara coinciden en varios puntos: todas se consideran víctimas al haber sido expuestas a una estrategia de indiferencia y de abandono; todas buscan que su dolor, que es humillación ante el desconocimiento de sus derechos, pena por las compañeras y los compañeros muertos e indignación por los años de privaciones y sufrimiento, debe ser compensado por el Estado o por un ente internacional que influya en las decisiones del gobierno colombiano; y finalmente, todas coinciden en que su historia es también la lucha del hospital por sobrevivir, por demostrar que no está parado en el tiempo, cerrado o amilanado y que, por el contrario, es en sí mismo la encarnación de la dignidad, el amigo que "nos enseña lo que es morir de pie".

A continuación propongo una lectura del caso del hospital centrándome en las codificaciones y moralidades inscritas en los artefactos que las trabajadoras cuidan, recolectan, 
producen y usan y en las prácticas cotidianas que operan como marcadores de diferenciación para demarcar los márgenes dinámicos y borrosos del Estado. Se trata, como plantea Viviana Zelizer (2009), de un "trabajo relacional" constante para separar los mundos aparentemente antagónicos de lo público y lo privado, lo doméstico y lo laboral, lo individual y lo colectivo. El caso del San Juan resulta bueno para pensar la producción de sentido en el marco de la economía moral contemporánea y la manera en que los actores (en este caso las mujeres trabajadoras del hospital) le hacen frente a la producción social de la indiferencia (Herzfeld, 1993) trazando narrativas, construyendo alianzas y empleando artefactos diversos para objetivar su historia y afirmar su humanidad.

\section{Una cocinera se toma el sindicato}

Gladys, trabajadora del Centro Hospitalario San Juan de Dios desde febrero de 1982, siempre lleva consigo una carpeta repleta de documentos que constituyen la prueba de una injusticia. Gladys recuerda que empezaron a pagarle su primer sueldo con seis meses de atraso, pero se quedó porque necesitaba el trabajo. Para ella la crisis ha sido una constante en la historia del hospital. Tan pronto ingresó al sindicato se dio cuenta que para quienes no eran profesionales era difícil participar en la toma de decisiones, entonces comenzó a organizar “a las niñas de la cocina" y a "las niñas de servicios generales" y en 1984 accedieron a varios cargos administrativos dentro de la organización. Los médicos dijeron que el sindicato había sido "tomado por cocineras" y, según Gladys, usaron todos los epítetos existentes para discriminarlas (por pobres y por mujeres), no obstante, ella siguió adelante y comenzó a trabajar en la Comisión de Reclamos. En este puesto, Gladys aprendió el significado de la expresión "llevar un debido proceso". Después pasó a la tesorería y finalmente llegó a la presidencia del sindicato.

En 1996 se firmó una convención colectiva que venció en 2016. La convención fue demandada por un grupo de directivos y trabajadores que pensaban que el hospital debía sufrir una "reestructuración administrativa" para seguir funcionado, pues no podía seguir "socorriendo miserables". Esta transformación organizacional tenía que ver básicamente con modificar los estatutos del San Juan para que se tornara una Empresa Social del Estado (ESE), como fueron llamados los hospitales púbicos luego de las reformas de "ajuste estructural" de la década de 1990 que privatizaron el sistema de seguridad social y salud en Colombia. Las ESE tenían que "modernizarse" y "armonizar su estructura a las nuevas leyes", habían que empezar a facturar, vender servicios y convertir las especialidades en "unidades empresariales". Esta reforma se materializó mediante la expedición del decreto 371 de 1998 del ministro de Protección Social y la suspensión de la nómina de los trabajadores para forzar la renuncia a la convención colectiva.

Entre 1996 y 1997, paralelamente a su actividad sindical, Gladys había empezado a trabajar en la Oficina de Cartera del San Juan, donde debía archivar y organizar las facturas enviadas por los proveedores a los cuales, pese a la crisis, se les seguía pagando. Gladys comenzó a analizar estos documentos y a encontrar irregularidades que convertía paulatinamente en denuncias. El nuevo puesto le permitió a Gladys estar al tanto de la actividad administrativa del hospital y sobre todo, "aprender a investigar mirando". En esa época empezó la presión 
para renegociar la convención de trabajo firmada en 1996 y Gladys, junto con un grupo de "base", comenzaron a hacer sus propios análisis. La hipótesis del "limbo" según la cual la reforma del hospital dejaría a los empleados sin patrón, empezaba a tomar fuerza y al tiempo, y con base estos hallazgos, comenzaron a planearse distintos tipos de acciones para hacer pública su indignación. Fue así como, el 16 de diciembre de 1999, las trabajadoras y trabajadores del Hospital San Juan de Dios se tomaron el Ministerio de Salud de Colombia. Llegaron poco a poco, vestidos con batas blancas para que fuesen identificados como médicos y ocuparon los distintos pisos del edificio. El objetivo era pelear el hospital, oponerse a las "unidades empresariales" que representaban la privatización progresiva del hospital y "exigirle al Estado" más presupuesto. A las tres de la mañana, luego de un intenso cubrimiento periodístico que hablaba de secuestro y de guerrilleros infiltrados, la Fuerza Disponible de la Policía bombardeó el edificio con gases lacrimógenos y sacó a la gente a la fuerza. Varias personas fueron arrastradas por la Carrera Séptima, mientras otras tantas comenzaron a gritar. La gente se levantaba y esgrimía banderas blancas, la idea era no salir hasta encontrar solución, pero ante la cantidad de hombres armados terminaron desistiendo y retornando al hospital.

El 20 de diciembre del 1999, las trabajadoras recibirían "un golpe muy duro": la "traición" de su principal aliada, representada en la salida de los médicos de la Universidad Nacional de Colombia. Cada vez quedaron menos personas, básicamente estudiantes, algunos "compañeros" de organizaciones sociales, las trabajadoras y un pequeño reducto de profesionales que operaba en urgencias. Antes de terminarse el año la Superintendencia de Salud dio potestad a la empresa de energía eléctrica de Bogotá, para suspenderle el servicio de luz por falta de pago. Este episodio es calificado por Gladys como un intento de homicidio: "cuando quitan la luz de cirugía casi mataron al hospital, con ese acto la Superintendente le indujo la muerte cerebral, lo puso a vegetar". Sin embargo, Gladys y sus compañeras, aprovechando las áreas que todavía tenían energía eléctrica, siguieron con su "análisis", implantaron una "olla comunitaria" y continuaron atendiendo pacientes. Al final quedaron 60 enfermos pues la mayoría fueron "sustraídos" por la Superintendencia Nacional de Salud.

Pese a la lucha de las trabajadoras y a un acuerdo marco firmado entre la Universidad Nacional, las directivas del sindicato, el director del hospital y la Procuraduría General de la Nación, la Superintendencia de Salud prohibió a todas las instituciones de salud del país que remitieran pacientes al San Juan. Entonces Gladys se fue para el césped y se puso a llorar de "pura impotencia". Estando allí un compañero que nunca había visto antes se le acercó y le preguntó qué le pasaba. Ella le respondió que había comenzado a hacer averiguaciones y que se había dado cuenta que el cambio de estatutos era ilegal porque el San Juan nunca había sido una "fundación". Entonces el muchacho le dijo: "Ya vengo, pero no llore". Y volvió con la copia de una ordenanza expedida por la Gobernación de Cundinamarca según la cual: "la Nación compra el terreno Molinos de la Hortúa [donde actualmente está ubicado el hospital] en el año 1906". El muchacho le llevó a Gladys el papel y le dijo: "usted puede tener la razón, arme su tesis". Ella se quedó leyendo y cuando volteó a mirar el compañero ya no estaba. Jamás lo volvió a ver.

Después del encuentro con el colaborador anónimo Gladys se fue para la Universidad a buscar a uno de los estudiantes de medicina que la apoyaba en ese entonces para buscar la fuente del documento. Este episodio es recordado especialmente por Gladys por tratarse de 
una muestra genuina de aquello que ella denomina "filosofía de la práctica" es decir, cuando un "intelectual se junta con el pueblo para investigar". Fue en esa búsqueda documental que esta pareja comenzó a reunir un inmenso número de papeles que sirvieron para demostrar que el hospital había sido adquirido por el Estado y que no era el producto de la donación de un particular. De esta colaboración nació la teoría de los "tres hospitales": el primero, de origen "privado", fundado en 1554 gracias a una donación del Arzobispo de Santa Marta; el segundo, de origen "público", mandado a hacer en 1723 por orden del Rey de España; y el último, una extensión republicana del segundo hospital, oficializada en 1911, cuando la Nación le cedió la administración (más no la propiedad) de los terrenos del hospital al Departamento de Cundinamarca. La teoría de los "tres hospitales" concluye que el San Juan es una institución pública de orden nacional, que no hay continuidad histórica ni institucional entre el primer y el tercer hospital. Por tal motivo los decretos que convirtieron al hospital en una "fundación", argumentando su origen privado, son ilegales. En 2005, el Consejo de Estado le dio la razón a Gladys y a otra trabajadora del San Juan, quienes interpusieron la "demanda de nulidad" de los decretos citados. Con esto la "Fundación San Juan de Dios" perdió su piso jurídico o, en otras palabras, dejó de existir legalmente. ${ }^{8}$

\section{El valor de los objetos}

Oscar es uno de los estudiantes que acompaña desde hace varios meses a las trabajadoras del San Juan y especialmente al grupo de Clara y otras personas que decidieron ocupar permanentemente su sitio de trabajo e irse a vivir allí con sus familias desde hace 16 años. El apoyo de Oscar y varios de sus compañeros ha sido importante durante las manifestaciones públicas de 2013 y 2014, caracterizadas por sacar a la calle diferentes objetos pertenecientes al hospital como una manera de innovar en la protesta y de llamar la atención de los transeúntes. El objetivo es, según comenta el estudiante, resignificar todos esos equipos que hoy están en desuso, que materialmente no tienen ningún valor, que pueden incluso ser considerados "chatarra", pero cuyo significado radica en los servicios que le prestaron a la comunidad en los tiempos en que el San Juan de Dios y el ya liquidado Instituto Materno Infantil atendían pacientes. Durante la última manifestación realizada el primer semestre de 2013 se exhibió una camilla perteneciente al Pabellón San Lucas. Como esa camilla hay todavía 1.200. Los estudiantes la recuperaron un poco, no demasiado, porque es importante que los signos del deterioro sigan visibles, y la llevaron a la marcha para decirle a la gente que “¡Sí hay camas!” y que “¡No tenemos que ir a los otros hospitales a decir que nos presten lechos para los pacientes". Ese día también llevaron a las calles una de las cunas del Materno Infantil, que contaba con más de novecientos cupos en sus épocas gloriosas. Estaba también la incubadora, que ahora es "solo la carcasa" porque después del proceso de privatización de ese hospital los equipos fueron desvalijados y vendidos por partes. Cada camilla tiene su propio "paciente" armado con materiales hallados en el propio hospital. Uno de ellos fue elaborado con fragmentos de piezas ortopédicas y tiene, además de una férula, sus respectivos líquidos intravenosos. Según Oscar todo esto lo han hecho "con las uñas", pero también con la confianza de que la gente que pase o participe en las marchas diga: "oiga, sí vale la pena rescatar al San Juan", se trata de 
"moverle la cabeza a la gente", de hacer que las personas entiendan que lo que ven es solo una muestra de las millones de cosas "que se están pudriendo".

En cierta ocasión, durante la época de la "liquidación" del San Juan, Clara y Oscar intentaron sacar una de las camillas y tuvieron problemas con el vigilante de turno, porque según él, dicho objeto estaba inventariado. Ante esto, Clara, cabeza visible de los trabajadores que habitan el centro hospitalario desde 1999, respondió de manera categórica: "como usted sabe eso no es así porque los lugares donde viven los trabajadores no están inventariados, por una cuestión de respeto y también para que no se roben las cosas". El vigilante se negó nuevamente alegando que cumplía órdenes de la "liquidadora". Clara y Oscar insistieron diciéndole al hombre que su labor era velar para que las cosas no se perdieran, que el propósito de las trabajadoras no era llevarse nada sino hacer una denuncia y que después de la marcha le devolverían la camilla intacta, junto con "el último gramo de mugre". Esta escena no era poco frecuente, de hecho, las bitácoras de celaduría, usadas para justificar posteriores acusaciones, hechas por la "liquidadora" y sus abogados en contra de las trabajadoras, registraban detalladamente todos los "movimientos inusuales" de estas mujeres, sus compañeros de causa y acompañantes frecuentes, muchos de los cuales portaban "sospechosas" mochilas y cabelleras largas.

Clara tiene una interpretación diferente a la de Gladys respecto al problema laboral y al estatus del hospital. Para ella la lucha consiste en arrebatarle el hospital al "Estado" o, como ella misma dice, al "gobierno de turno", es decir, no tiene sentido pelear porque el San Juan sea público, puesto que la situación actual es precisamente consecuencia de la intervención de representantes directos e indirectos del "Estado" como el antiguo Gobernador de Cundinamarca y la propia "liquidadora". Desde este punto de vista, no importa si el hospital es declarado privado porque a la larga lo fundamental es que continúe prestando sus servicios al pueblo. No es un debate ideológico, ni político, afirma Clara, se trata de un hospital que debe seguir funcionando, de una entidad que está para salvar vidas y la vida no es ni privada ni pública, "es vida y punto". De ahí el rechazo de Clara y varios de sus compañeros quienes, valga decirlo, se niegan a ser identificados como miembros de "la base", de aceptar propuestas como la de la Secretaría de Salud del Distrito, entidad que, según Clara, quiere usar las instalaciones del hospital sin comprometerse con la cancelación de la deuda de los trabajadores, ya que "no va a pagar nada más allá de lo que obliga la SU". ${ }^{9}$ Aquí cobra sentido la idea de trabajar con los objetos vetustos del San Juan, pues se trata apenas del primer paso de un plan más ambicioso consistente en hacer que el pueblo, no el "Estado", se apropie del hospital. Por eso se reúnen todos los viernes, en un encuentro abierto al público, para exhibir los objetos, algunos de los cuales han sido recuperados, como por ejemplo una cabina que llamaron "Fénix" y que poco a poco han ido convirtiendo en un "consultorio", representando así el primero de varios espacios que constituyen el proyecto de reactivación del primer piso del hospital hecho por el pueblo.

En esta nueva inflexión de la causa, el papel de las instituciones de la administración pública sería doble. Por un lado "reactivar" el San Juan, devolviéndole lo que siempre ha sido suyo, o sea, los bienes que le fueron arrebatados y que le permitirían convertirse en un ente autosustentable; por otro, cumplir con la responsabilidad de solucionar el conflicto laboral. Este énfasis es central para este colectivo de trabajadoras y estudiantes, quienes sostienen que 
la lucha del hospital y la lucha de los empleados está relacionada pero no es la misma cosa. Por esta razón, Clara corrige pacientemente a todos los colaboradores que, aunque con buenas intenciones, insisten en que el trabajo con los objetos equivale a construir un "museo de la memoria" del San Juan. "Voy aclararte una cosa con todo respeto", le dijo la trabajadora a un investigador que le preguntó si esta colección de objetos era un museo, "no es museo, es una exposición para darle a conocer a la opinión pública que aquí hay cosas valiosas. Que unas se las han robado y que otras las tenemos por sentido de pertenencia para que las conozcan." Así que las camillas, incubadoras, sillas de ruedas, instrumental quirúrgico y materiales ortopédicos en desuso son, según Clara: "las boronas que nos ha dejado el gobierno nacional, lo que no se ha podido desmantelar". Cada vez que algún incauto le hace la misma pregunta, Clara reitera que esos objetos son exhibidos para que el pueblo se acerque y entienda que el valor de esas cosas está en su relación con "la vida", porque muchas personas creen "que lo que vale es el San Juan", pero en verdad lo más importante es "la vida de cada paciente que no se ha podido salvar" y "de cada persona que ha muerto y enfermado en esta lucha". Para este grupo de trabajadoras y sus aliados, los objetos representan el costo de la causa, por tanto, ni tienen precio, ni están anclados en el pasado, por el contrario, son totalmente vigentes: si no fueran lo que son, si no estuvieran como están, no adquirirían sentido en su universo simbólico. El tema de los objetos adquiere así un lugar espacial en la construcción de la causa que venimos describiendo: al decir que unas camillas abandonadas por la decidía del "Estado" y roídas por el paso del tiempo no pueden hacer parte de un inventario, al declarar invaluables ciertos objetos que a los ojos de un observador externo parecen simple "chatarra", al oponer la dimensión performativa de esos materiales a la lectura museológica, Clara y sus amigos están hablando de una manera particular de producción de valor que parece responder a un entrelazamiento afectivo, económico y moral.

\section{Cuidando y queriendo al San Juan}

Ligia estudió enfermería en la Universidad del Rosario, venía de una familia acomodada de Boyacá y viajó a Bogotá buscando realizar estudios profesionales. Cuando, en 1998 dejó de recibir su salario como trabajadora del San Juan, tuvo que afrontar una dura crisis que afectó su vida familiar y profesional. Al ver como sus ahorros disminuían vertiginosamente, decidió pasar hojas de vida a otras instituciones de salud, entregó un total de 189 currículos pero en ningún sitio fue aceptada porque, según ella, era víctima de estigmatización al haber sido trabajadora del San Juan. Ligia y varias de sus compañeras, también profesionales, afrontaron la desestabilización producida por la crisis de diferentes maneras: se agruparon para continuar trabajando en el hospital, para sostenerse económicamente y para "olvidar la realidad" como ella misma dice. Nunca han dejado de firmar la lista de asistencia ni de pasar la solicitud de sus vacaciones y prestaciones sociales a la oficina de personal, puesto que se consideran trabajadoras activas.

A partir de la salida de los médicos de la Universidad Nacional, ella y varias de sus compañeras, especialmente enfermeras, terapeutas y otras "profesionales del cuidado" comenzaron a organizarse para que el hospital siguiera funcionando, pues nunca cerró, por el contrario, espera a sus pacientes y estudiantes con los "brazos abiertos". En los primeros 
años, cuando había todavía 1.600 trabajadores, fueron varias las estrategias adoptadas por ellas: instauraron las "caravanas" (convoyes compuestos por dos ambulancias que hacían las veces de camión con las cuales recorrían las plazas de mercado en busca de donaciones para preparar los alimentos de pacientes y empleados); realizaban diariamente jornadas de aseo y mantenimiento de los edificios y recogían dinero en fondos de empleados y sindicatos para comprar gasolina y pagar los gastos de administración más apremiantes. Según Ligia con todas estas actividades lograban mantener el hospital y demostrarle a la Superintendencia de Salud que podían seguir operando a pesar del abandono. Entre tanto, varios de los médicos de planta comenzaron también a reunirse pero "con otro enfoque": no buscaban salvar al hospital, afirma Ligia, sino salvarse ellos mismos escribiendo cartas en donde decían que a partir de la fecha no se responsabilizaban con lo que ocurriera con los pacientes.

Cuando la Superintendencia de Salud impidió la remisión de pacientes, Ligia y sus compañeras decidieron seguir atendiendo. El 21 de noviembre de 2002 estrenaron una nueva estrategia que denominaron "Jornadas de Atención Médica" que consistió en prestar servicios de consulta externa el último domingo de cada mes, de 7 de la mañana a 8 de la noche. Ligia recuerda que ella y las demás profesionales alistaban y limpiaban la planta física y todos los implementos, previendo las visitas de las entidades sanitarias. Como no tenían luz, usaban una planta eléctrica que prendían desde el día anterior y alimentaban con el crudo donado "por los compañeros del sindicato de Ecopetrol". Además de prestar sus servicios como profesionales de la salud, este grupo de mujeres se esmeraba por mantener pulcro el hospital, aseaban todos los pisos del edificio principal y las instalaciones de urgencias, hasta que un día de 2006 la liquidadora cambió las cerraduras y no las dejó entrar más.

Ligia y Julia, su colega más querida, tomaron la decisión de averiguar lo que estaba pasando "verdaderamente" en el San Juan cuando, en 2000, visitaron el Centro Administrativo Distrital y encontraron una maqueta con la proyección de la renovación urbana del centro de Bogotá. En esa visión de futuro, el San Juan aparecía proyectado como un garaje para estacionar la flota de buses del sistema de transporte masivo. Ante la indignación de ver convertido su hospital y parte de su vida en un estacionamiento decidieron tomar medidas, pero esta vez buscando apoyo político. Ligia cuenta que se dirigieron al Congreso de la República y su consigna comenzó a tomar otros matices cuando consiguieron despertar el interés de algunos congresistas. Fue así como apareció la idea del "patrimonio" como una nueva estrategia de lucha. ${ }^{10}$ Para evitar la demolición, para impedir que el San Juan se transformara en ese paisaje desértico y plano que habían visto en miniatura, Ligia y sus compañeras emprendieron una nueva campaña para convertir el San Juan en patrimonio histórico de la Nación. Y ciertamente esa nueva herramienta discursiva parecía describir muy bien la situación de las trabajadoras pues, como ellas mismas dicen, patrimonio "es todo, tanto el estilo francés de los edificios, como los cuadros de Arce y Ceballos y los saberes de las personas que allí trabajamos". Fue así como consiguieron el apoyo de una senadora del Partido Liberal, procedimiento duramente criticado por el sindicato de trabajadores afín a los partidos de izquierda, que denominó "iniciativa burguesa" al intento por constituir una ley de patrimonio para salvar al San Juan. Al mismo tiempo, y como complemento a este trabajo político, Ligia y sus compañeras decidieron realizar su propia investigación acerca de la historia del San Juan y para esto construyeron su propio archivo, empezando a buscar y almacenar documentos de todo tipo. 
En 2002 el Hospital San Juan de Dios fue declarado Patrimonio Cultural de la Nación. Esto convertía a la institución en "un lugar interesante desde el punto de vista de la política cultural" y daba a las trabajadoras nuevas herramientas para defender su causa, pues los predios que componen el San Juan deben ser preservados para las futuras generaciones y no pueden, bajo ningún motivo, ser objeto de enajenación o intercambio comercial. Con la declaración de Patrimonio, Ligia y sus compañeras comenzaron a trabajar con mayor frecuencia en el ámbito de la "cultura". Participaban en los Consejos de Cultura de la Alcaldía, donde nació la idea de crear un programa para que los ciudadanos pudieran conocer el legado del San Juan, aquello que Ligia denomina en términos técnicos "lo tangible y lo intangible" o en otras palabras, los saberes y las cosas que constituyen el patrimonio. Por este camino, este grupo de mujeres, provenientes de profesiones relacionadas con la salud y el cuidado, comenzarían a formarse como "guías turísticas". Terminado el curso, las profesionales estaban listas para implementar un nuevo programa patrocinado por la Alcaldía Mayor de Bogotá que ellas mismas llamaron "Siga, esta es su casa", que tenía como objetivo promover el San Juan como un sitio de interés histórico. Cuando las personas llegan, ellas se presentaban como trabajadoras del hospital identificándose con su nombre y su respectivo cargo. A continuación se les recalcaban a los visitantes que se encontraban en el "mejor hospital de Suramérica", que en la actualidad estaba atravesando por una grave crisis, pero que continuaba vivo, que era solo cuestión de "ponerle las sábanas" a las camas para que comenzara a funcionar de nuevo.

Entre 2006 y 2009, luego de que comenzara el proceso de "liquidación" y les fuera negada la entrada a la mayoría de los espacios que comprendían el recorrido turístico, Ligia y sus compañeras cambiaron nuevamente de estrategia. Esta vez decidieron ir a las universidades a contar la historia, especialmente a la Universidad Nacional, la antigua aliada. Las profesionales eran invitadas a distintas cátedras y allí les recalcaban a los estudiantes que el Instituto Materno Infantil (IMI), el San Juan y la Universidad eran una sola cosa, algo que no podía disolverse, pero que la Universidad había actuado como una mujer coqueta cambiando al San Juan por 168 maridos (referencia a los nuevos lugares de práctica), mientras que "el señor antiguo, viejo, el mayor de todos los hospitales", la seguía esperando, "con la casa lista, porque nunca la cambió por otra".

\section{Consideraciones finales}

Para finalizar quisiera presentar algunas consideraciones sobre la teoría nativa construida por las trabajadoras según la cual el San Juan es "un sujeto de derechos". Para esto será necesario contar brevemente el devenir del conflicto entre las trabajadoras y el "Estado" y luego explicar desde una perspectiva antropológica por qué el hospital puede ser descrito como una "persona moral". Esto implica una revalorización del papel de los "objetos" en la descripción etnográfica, mostrando su capacidad de agencia y su participación activa en la composición de universos sociales. Al final, espero demostrar que los argumentos aquí presentados permiten señalar la necesidad de explorar las dimensiones morales y existenciales de las luchas de colectivos y movimientos sociales, yendo más allá de la imposición semántica y epistemológica que implica reducir los resultados de una causa a los códigos impuestos por el "discurso recto" del derecho. 
Nuevos conflictos aparecieron con la llegada de la contratista de la Gobernación de Cundinamarca para liquidar la "Fundación San Juan de Dios", proceso que según el tiempo del "Estado" comenzó en 2006 y terminó en mayo de 2013: hizo sellar la mayor parte de los edificios del hospital; entorpeció las jornadas de atención médica; subastó una gran cantidad de equipos clínicos y de infraestructura; denunció a las trabajadoras ante las autoridades de policía por haber "invadido" el hospital y usado los edificios como vivienda en detrimento del patrimonio histórico y cultural de la Nación y de la salud de sus familias y mandó fumigar la huerta comunitaria con glifosato, alegando que "los invasores" constituían un "reducto guerrillero" y mantenían "cultivos ilícitos" en las zonas verdes de la institución. En 2015, la Alcaldía afirmó que compraría el San Juan para reactivarlo y convertirlo en un centro médico de "cuarto nivel", sin embargo la hipótesis de la "fundación ilegal", que se tornó verdad jurídica, impide la venta, pues el hospital legalmente no tiene dueño. Entre tanto, algunas trabajadoras siguen avivando la causa e idean nuevas acciones para defender el hospital: continúan oponiéndose a varios agentes de "Estado" que insisten en despojarlas de su forma de autorreconocimiento y en demostrar que ellas no son parte del San Juan; ocupan sus puestos de trabajo; reclutan estudiantes e investigadores para la causa; recorren, con quien quiera acompañarlas, los edificios ubicados en diferentes puntos de Bogotá que pertenecen históricamente al hospital; gestionan audiencias públicas en el Congreso de la República y, sobre todo, hacen historia, articulando en sus narrativas afectos, y política, juntando las pruebas de una injustica, los artefactos que les permiten hacer verosímil su existencia como agentes activos del mundo laboral y demostrar que el San Juan es un "sujeto de derechos".

La fabricación del universo social en el cual las ideas y prácticas de las trabajadoras cobran sentido es también la fabricación del San Juan como persona moral. El hospital es más que un conjunto arquitectónico, es un idioma activado por los actores sociales para afirmar su humanidad, un vehículo para la elaboración simbólica de una causa y un medio para entender la construcción de colectividades a partir de gramáticas emocionales y la articulación de experiencias subjetivas y reivindicaciones políticas (Fernández, 2011) o, en otras palabras, el paso de la existencia serial (individual, fragmentada y egoísta) a la existencia colectiva y solidaria (Bourdieu, 1984). El San Juan es también una entidad dinámica antropomorfizada, construida por medio de analogías biomédicas, orgánicas, escatológicas y emocionales (muere de pie, se queda sin oxígeno, cae en cuidados intensivos, se levanta, abre los brazos, espera, es traicionado), es decir, con cuerpo y género, que construye su propia espacialidad en la circulación de personas y artefactos diversos (legados, testamentos, escrituras públicas, cédulas reales, instrumental quirúrgico, consultorios improvisados, predios robados por abogados deshonestos, terrenos usurpados por agentes de la administración pública); que producen demarcaciones y circunscripciones morales del espacio. El San Juan está dotado de agencia y es, por tanto, mucho más que un "objeto", es un ser amado y cuidado por este grupo heterogéneo de mujeres que compila sus memorias, cuenta su historia y le otorga valor a sus pertenencias.

Los documentos son la sangre que fluye a través de la persona moral producida en la lucha de las trabajadoras del San Juan. No es posible entender esta causa sin prestar atención a la circulación de estos artefactos y a la manera por medio de la cual son usados para demostrar el parentesco del San Juan con el "Estado". Estos papeles pueden ser descritos como superficies 
(Feldman, 1991), sitios, estadios, moldes, sobre los cuales la historia es construida como objeto cultural, o para este caso, artefactos sobre los cuales es posible rastrear los efectos teleológicos y de pretendida continuidad de esa abstracción mistificada que llamamos "Estado". En ese sentido, Gladys y Ligia son historiadoras nativas que describen la vida social del San Juan ampliando y reduciendo la exterioridad del "Estado", construyendo sus propias narrativas y temporalidades, organizando elementos heterogéneos, formulando tramas coherentes a partir de colecciones fragmentadas de papeles (Ortega, 2008; Lowenkron, Ferreira, 2014) firmados y sellados por actores diversos que representan al gobierno legítimo de los bienes públicos. No hay que olvidar que estas prácticas están sometidas a la verificación burocrática y son constituyentes de los procesos de formación de "Estado", única unidad moral representativa, obligada a purificar, responder y ajustar cuentas con el pasado (Borneman, 1997).

Gladys, Ligia y Clara, y en un sentido más amplio los actores sociales que le otorgan sentido a esa trama relacional que llaman el "San Juan", nos señalan caminos para entender la construcción de dimensiones morales forjadas a partir de un conflicto por el reconocimiento de derechos. Se trata de un conflicto vivo, configurador de mundos sociales, de comunidades emocionales que solo puede entenderse a partir de un análisis procesual y simbólico de esas verdades en disputa denominadas derechos, de la descripción de los procedimientos mediante los cuales los actores buscan fijar cierto tipo de significados a partir de una causa común, en este caso, la condición de "trabajadoras del San Juan", negada por la racionalidad jurídica y la imposición violenta del tiempo del "Estado". A pesar de la insistencia institucional por ubicar la "vida laboral" de estas mujeres en el pasado, tal como ocurre con la "vida útil" de los objetos que componen el "inventario" del San Juan, y aunque el óxido imprima inexorablemente el paso del tiempo en los antiguos equipos radiológicos y quirúrgicos, las trabajadoras nos recuerdan que los artefactos que componen el hospital tienen agencia, que el "patrimonio" es presente y futuro, que está impreso en objetos ordinarios y que su valor es inconmensurable pues representa el costo (físico y metafísico) que han tenido que pagar por defender "la salud del pueblo". Para las trabajadoras, el patrimonio es lucha y no objeto museológico, es por tanto invaluable, al igual que su sufrimiento, al igual que las vidas de los compañeros y compañeras fallecidos esperando los salarios atrasados.

Noventa trabajadoras y trabajadores han muerto desde el inicio de este conflicto. Algunas personas fueron indemnizadas y abandonaron la causa, otras, desesperadas, firmaron acuerdos con los liquidadores de turno. Las historias de Gladys, Ligia y Clara. presentadas aquí, muestran que las colectividades son profundamente heterogéneas ( $\mathrm{y}$ en ese sentido términos como identidad o comunidad no ayudan a entender la complejidad de sus asociaciones), que sus conexiones son siempre contingentes y parciales, tan frágiles que pueden romperse en cualquier momento, pero a la vez tan fuertes que pueden resistir el paso del tiempo gracias un costo enorme de energía, a un constante trabajo relacional (Zelizer, 2009) para reivindicar una condición existencial. De ahí el valor de su trabajo, que va más allá de una definición estrictamente jurídica o materialista, su lucha consiste en contradecir, día tras día, la tesis de abogados y "liquidadores" según la cual ellas: "no trabajan porque no ejercen funciones". 


\section{AGRADECIMIENTOS}

Este artículo es fruto de un trabajo conjunto de investigación de la Universidad Nacional de Colombia y un grupo de trabajadoras del Hospital San Juan de Dios, realizado entre 2013 y 2014, en el cual participaron Margarita Castro, Edelmira Arias, Susana Fergusson, Sara Zamora, Jenny Fonseca y Ramiro Borja. Agradezco también a Adriana Vianna, César Abadía, Moacir Palmeira, Graziele de Lima y Johanna Salazar por sus oportunas críticas y comentarios.

\section{NOTAS}

${ }^{1}$ La lucha comenzó en el gobierno de Andrés Pastrana (1998-2002) y prosiguió durante la presidencia de Álvaro Uribe (2002-2010) y Juan Manuel Santos (2010 - hasta la fecha).

${ }^{2}$ Como se verá más adelante el año de fundación del San Juan es controversial. En su trabajo monumental sobre la historia de la enfermedad, la pobreza y la muerte en Bogotá, Estela Restrepo (2013) afirma que el hospital fue creado en 1635.

${ }^{3}$ Para una discusión sobre las consecuencias de las reformas neoliberales al sistema de salud en Colombia, desde una perspectiva etnográfica, véase el trabajo pionero de César Abadía-Barreto $(2011,2015)$ y varios investigadores del Grupo de Antropología Médica Crítica de la Universidad Nacional de Colombia (Ariza, Abadía-Barreto, Pinilla, 2013; Melo, 2013).

${ }^{4}$ La convención colectiva, firmada en 1996, tenía una vigencia de veinte años. Allí se pactó un aumento del salario y la permanencia de la pensión de jubilación después de 20 años de trabajo. También se subrayó la necesidad de continuar laborando con contratos a término indefinido y de mantener el carácter "estatal" del hospital. Se trataba de preservar los derechos laborales básicos amenazados por las reformas neoliberales implementadas a partir de 1993. Estas reformas incluyeron la "flexibilización" de los contratos de trabajo y la privatización de los fondos de pensiones y del sistema de seguridad social en salud.

${ }^{5}$ Como estrategia político-narrativa, he optado por proteger la identidad de las trabajadoras y mezclar varias historias bajo un solo nombre, intentando respetar las distintas perspectivas y posiciones que han generado conflictos internos y luchas comunes.

${ }^{6}$ Referencia al testamento del millonario José Joaquín Vargas, quien le heredó al hospital los terrenos correspondientes a la Hacienda El Salitre. Estos terrenos fueron divididos y negociados por la Gobernación de Cundinamarca como si fueran suyos. Parte del dinero se usó para "sanear" las finanzas del hospital y conformar "La Fundación" en la década de 1970, el resto se puso en un Fideicomiso y nunca fue invertido en el hospital (Malaver, 26 oct. 2012).

${ }^{7}$ Hay una controversia histórica entre Gladys y otras "historiadoras nativas" del grupo de Ligia, quienes argumentan continuidad entre los tres hospitales con el objetivo de pleitear un legado aún mayor.

${ }^{8}$ No obstante, la demanda de nulidad tuvo un efecto adverso. Al quedar anulada la Fundación, el gobernador de Cundinamarca insistió en que había que "volver" a 1978 cuando el hospital era "propiedad" del Departamento. Este argumento jurídico abrió la puerta para la "liquidación" del hospital en 2006.

${ }^{9}$ Referencia a la Sentencia de Unificación 484 de 2008 emitida por la Corte Constitucional.

${ }^{10}$ Sobre las relaciones entre el proceso de patrimonialización del hospital como un proceso de "resistencia" puede consultarse el trabajo del politólogo Daniel Amézquita (2013). Sobre la militancia de las trabajadoras en los primeros años de la década de 2000, véase la investigación de Humberto Cárdenas (2004).

\section{REFERENCIAS}

ABADÍA-BARRETO, César.

The transformation of the value of life: dispossession as torture. Medical Anthropology, v.34, n.5, p.1-18. 2015.

ABADÍA-BARRETO, César et. al.

Algunas violencias del capitalismo en Colombia:

Dispositivos de tortura y autodestrucción".

Maguaré, n.25, p.203-240. 2011.
AMÉZQUITA, Daniel.

La apropiación de la memoria del San Juan de Dios. [Documento inédito]. 2013.

ARIZA, Katherine; ABADÍA-BARRETO, César; PINILLA, María J.

La praxis del Estado colombiano en la eliminación del Instituto de Seguros Sociales y del Instituto Materno Infantil. In: Abadía- 
Barreto, C. et al. (Ed.). Salud, normalización y capitalismo en Colombia. Bogotá: Universidad Nacional de Colombia. 2013.

BOLTANSKI, Luc.

La souffrance à distance: morale humanitaire, médias et politique. Paris: Éditions Métailie. 1993.

BORNEMAN, John.

Settling accounts: violence, justice and accountability in postsocialist Europe. Princeton: Princeton University Press. 1997

BOURDIEU, Pierre.

La délégation et le fetichisme politique. Actes de la Recherche en Sciences Sociales, n.52-53, p.49-55. 1984.

CÁRDENAS, Humberto.

Hospital San Juan de Dios: voces pieles y resistencias. Bogotá: Asociación de Trabajo Interdisciplinario. 2004.

CARDOSO DE OLIVEIRA, Luís Roberto.

A dimensão simbólica dos direitos e a análise dos conflitos. Revista de Antropologia - USP, v.53, n.2, p.451-473. 2010.

DAS, Veena.

Sufrimientos, teodiceas, prácticas disciplinarias y apropiaciones. In: Ortega, F. (Ed.). Veena Das: sujetos de dolor, agentes de dignidad. Bogotá: Universidad Nacional de Colombia. 2008.

DAS, Veena; POOLE, Deborah.

State and its margins comparative ethnographies. In: Das, V.; Poole, D. (Ed.). Anthropology in the margins of the State. Santa Fe: School of American Research Press. 2004.

FELDMAN, Allen.

Formations of violence: the narrative of the body and political terror in Northern Ireland. Chicago: The University of Chicago Press. 1991.

FERNÁNDEZ, María.

Além da racionalidade: o estudo das emoções como práticas políticas. Maná, v.17, n.1, p.41-68. 2011.

FOUCAULT, Michel.

Seguridad, territorio, población. México: Fondo de Cultura Económica. 2007.

HERZFELD, Michael.

The social production of indifference: exploring the symbolic roots of Western bureaucracy. Chicago: The University of Chicago Press. 1993.
JEGANATHAN, Pradeep.

Checkpoint anthropology identity and the state. In: Das, V.; Poole, D. (Ed). Anthropology in the margins of the State. Santa Fe: School of American Research Press. 2004.

JIMENO, Myriam.

Emoções e política: a vítima e a construção de comunidades emocionais. Maná, v.16, n.1, p.99121. 2010.

LOWENKRON, Laura; FERREIRA, Leticia. Anthropological perspectives on documents: ethnographic dialogues on the trail of police papers. Vibrant, v.11, n.2, p.75-111. 2014.

MALAVER, Carlos.

La herencia que no pudo salvar el San Juan de Dios. El Tiempo, Bogotá, p.13. 26 jun. 2012.

MELO, Marco.

"Quítate tú pa' ponerme yo": un análisis prospectivo de Ciudad Salud desde una perspectiva marxiana. In: Abadía-Barreto, C. et al. (Ed.). Salud, normalización y capitalismo en Colombia. Bogotá: Universidad Nacional de Colombia. 2013.

ORTEGA, Francisco

Rehabilitar la cotidianidad. In: Ortega, F. (Ed.). Veena Das: sujetos de dolor, agentes de dignidad. Bogotá: Universidad Nacional de Colombia. 2008.

RESTREPO, Estela.

El Hospital San Juan de Dios 1635-1895: una historia de la enfermedad, pobreza y muerte en Bogotá. Bogotá: Universidad Nacional de Colombia. 2013.

RILES, Annalise.

Introduction: in response. In: Riles, Annalise. Documents: artifacts of modern knowledge. University of Michigan Press. 2006.

VIANNA, Adriana.

O fazer e o desfazer dos direitos: experiências etnográficas sobre política, administração e moralidade. Rio de Janeiro: Epapers/Laced. 2014.

WEBER, Max.

The sociology of religion. Boston: Beacon Press. 1993.

ZELIZER, Viviana.

La negociación de la intimidad. Buenos Aires: Fondo de Cultura Económica. 2009. 